Available online on 15.02.2019 at http://jddtonline.info
(c) 2011-18, publisher and licensee JDDT, This is an Open Access article which permits unrestricted
non-commercial use, provided the original work is properly cited

Open $\odot$ Access

Research Article

\title{
Reverse Phase-High Performance Liquid Chromatography method development and validation for estimation of efavirenz by Quality by Design approach
}

\author{
Waghmare Santosh A a , Kashid Arun M b \\ a JSPM's Charak College of Pharmacy and Research(Affiliated to Savitribai Phule Pune University), Wagholi, Pune- 412207, India \\ b Department of Pharmaceutical Chemistry, Sinhgad Institute of Pharmacy, Narhe, Pune 411041, Maharashtra, India
}

\begin{abstract}
Quality by design (QbD) refers to the achievement of certain predictable quality with desired and predetermined specifications. A very useful component of the QbD is the understanding of factors and their interaction effects by a desired set of experiments by using software (design expert 8). The present study describes the development of a comprehensive science and risk based HPLC method which is given by design expert 8 and subsequent validation for the analysis of Efavirenz active pharmaceutical ingredient (API) using a quality by design approach. An efficient experimental design based on systematic scouting of all four key components of the RP-HPLC method (column, pH, mobile phase and flow rate) is presented. The described method was linear. $\mathrm{R}^{2}=0.9998$. The precision, ruggedness and robustness values were also within the prescribed limits ( $<1 \%$ for system precision and $<2 \%$ for other parameters). Chromatographic peak purity results indicated the absence of co-eluting peaks with the main peak of Efavirenz. The proposed method can be used for routine analysis of Efavirenz in quality control laboratories.
\end{abstract}

Keywords: Quality by design, HPLC, Efavirenz, design expert 8.

Article Info: Received 16 Jan 2019; $\quad$ Review Completed 10 Feb 2019; $\quad$ Accepted 12 Feb 2019; Available online 15 Feb 2019

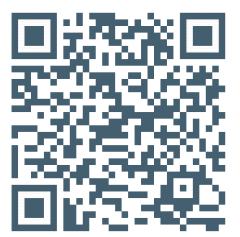

Cite this article as:

Waghmare SA, Kashid AM, Reverse Phase-High Performance Liquid Chromatography method development and validation for estimation of efavirenz by Quality by Design approach, Journal of Drug Delivery and Therapeutics. 2019; 9(1-s):319-330 DOI: http://dx.doi.org/10.22270/jddt.v9i1-s.2357

Mr. Santosh A. Waghmare, JSPM's Charak College of Pharmacy and Research (Affiliated to Savitribai Phule Pune University), Wagholi, Pune412207, India

\section{INTRODUCTION}

\subsection{Introduction to Drug Molecule}

Chemically efavirenz is (4S)-6-chloro-4-(2cyclopropylethynyl)-4-(trifluoromethyl)-2,4-dihydro-1H3,1-benzoxazin-2-one. It has molecular formula of $\mathrm{C}_{14} \mathrm{H}_{9} \mathrm{ClF}_{3} \mathrm{NO}_{2}$. It is crystalline powder having white or slightly yellowish colour. The melting point of Efavirenz is ranging from $136.0^{\circ} \mathrm{C}$ to $141.0^{\circ} \mathrm{C}$. Efavirenz is a nonnucleoside reverse transcriptase inhibitor (NNRTI). Efavirenz is directly connected to the enzyme and blocks the enzyme catalytic site. It is used as a part of highly active antiretroviral therapy for the treatment of human immunodeficiency virus. The drug is used in combination with other anti-retroviral agents for the treatment of HIV-1 infection in children's and adults. The usual dosage of efavirenz is $600 \mathrm{mg}$ per day. It is usually taken on empty stomach at bedtime to reduce neurological and psychiatric adverse effects. Structure of efavirenz is given in Figure no $1 .^{1}$

1.2 Quality by Design (QbD) is a concept first outlined by Juran in various publications. Juran believed that quality could be planned and that most quality crises and problems relate to the way in which quality was planned in the first place. Experimental design (DoE), as a tool of QbD, can be defined as the strategy for setting up experiments in such a manner that the information required is obtained as efficiently and precisely as possible. Experimental design (DoE) is a powerful technique used for exploring new processes, gaining increased knowledge of the existing processes and optimizing these processes for achieving world-class performance. Experimentation is performed to determine the relationship between factors acting on the response. ${ }^{2}$<smiles>O=C1Nc2ccc(Cl)cc2[C@@](C#CC2CC2)(C(F)(F)F)O1</smiles>

Figure 1: Chemical structure of efavirenz 
The choice of DoE is very important for the success of any industrial experiment. The proper selection was based on the process knowledge and depended on the following factors:

- Number of factors and interactions to be studied

- Complexity of using each design

- Statistical validity and effectiveness of each design

- Ease of understanding and implementation

- $\quad$ Nature of the problem

- Cost and time constraints

The potential benefits of using a DoE rather than using traditional "one factor at a time" experimentation are summarized below:

- Improved process yield and stability

- Improved profits

- Improved process capacity

- $\quad$ Reduced process variability

- $\quad$ Reduced manufacturing costs

- Reduced process design and developing time. ${ }^{2}$

1.3 Full three-level factorial designs by Design expert 8 Software.

Full three-level factorial design is an experimental matrix that has limited application in RSM when the factor number is higher than 2 because the number of experiments required for this design (calculated by expression $\mathrm{N}=3 \mathrm{k}$, where $\mathrm{N}$ is experiment number and $\mathrm{k}$ is factor number) is very large, thereby losing its efficiency in the modelling of quadratic functions. Because a complete three-level factorial design for more than two variables requires more experimental runs than can usually be accommodated in practice, designs that present a smaller number of experimental points, such as the Box-Behnken, central composite, and Doehlert designs, are more often used. However, for two variables, the efficiency is comparable with designs such as central composite. The majority of applications of three-level factorial designs are in the area of chromatography. $2,3,4,5,6$.

\section{MATERIAL AND METHODS}

\subsection{Apparatus}

A UV- vis Spectrophotometer (Jasco/ v-630), was used with quartz cells of $10 \mathrm{~mm}$ path length; HPLC (Jasco 12000 series); Column Symmetry C-18, 4.6*250mm, $5 \mu \mathrm{m}$ particle size (Waters); analytical balance (Contech, CB-50 series); ultrasonicator cleaning bath ( spectra lab / UCB-400); filter papers $0.45 \mu \mathrm{m}$.

\subsection{Materials}

Efavirenz reference standard was obtained as gift sample from Lupin Ltd. Aurangabad, Maharashtra, India and formulation was procured from the market. Ammonium formate buffer of research grade was obtained from Fine chem. Industries Ltd. Mumbai. Acetonitrile of HPLC grade was used for research from Merck Pvt. Ltd. Mumbai.

Stock solution of Efavirenz $1000 \mu \mathrm{g} / \mathrm{ml}$ was prepared by using $10 \mathrm{mg}$ of Efavirenz in $10 \mathrm{ml}$ of methanol.

\subsection{Chromatographic System}

Analysis was carried on Symmetry C-18 4.6*250mm, $5 \mu \mathrm{m}$ particle size column at $292 \mathrm{~nm}$. The samples were introduced through a Rheodyne injector valve with $20-\mu \mathrm{l}$ sample loop. The mobile phase consisted of acetonitrile and ISSN: 2250-1177 ammonium formate $(72: 28, \mathrm{v} / \mathrm{v})$, filtered through a membrane filter $(0.45 \mu)$, degassed in ultrasonic bath, and pumped at a flow rate of $1.0 \mathrm{ml} / \mathrm{min}$.

\section{RESULTS AND DISCUSSION}

\subsection{Design of Experiment}

Two Columns have used
$\checkmark$ Column C8
$\checkmark$ Column C18

\subsubsection{3 level Factorial design (Miscellaneous Factorial design)}

Selected Factorial design was miscellaneous Factorial design due to it has flexibility to change/add/delete any parameter at any time when our experiment is going on.it provide facility to give standard run at one time at only one mobile phase. ${ }^{6}$

\subsubsection{There are selected four mobile phase}

\author{
$\checkmark$ Acetonitrile : Water \\ $\checkmark$ Acetonitrile: Ammonium format buffer \\ $\checkmark$ Methanol : Water \\ $\checkmark$ Methanol : Ammonium format buffer.,3,4,5,6
}

3.1.3 Miscellaneous Factorial design can pick up one mobile phase. So selected each mobile phase one by one.

$\checkmark$ Acetonitrile : Water

$\checkmark$ Change pH Range: 3-5

$\checkmark$ Change Mobile phase proportion Range: 60-80\% (Consider Acetonitrile)

$\checkmark \quad$ Change flow rate range: $0.8-1.2$

When all above ranges put in Miscellaneous Factorial design. It gave 27 run at different $\mathrm{pH}$, Mobile phase proportion and flow rate.

Followed same procedure for each mobile phase. That is for column C-8 has four mobile Phase and column C-18 has four mobile Phases with 27 run for each mobile phase. Each column has its 108 run. After completion of all trails software give one optimize best value for each column. Optimization means finding an alternative with the most cost effective or highest achievable performance under the given constraints, by maximizing desired factors and minimizing undesired ones. In comparison, maximization means trying to attain the highest or maximum result or outcome without regard to cost or expense.

Miscellaneous factor design was utilised for method development in order to evaluate the effects of amount of buffer, buffer $\mathrm{pH}$ and flow rate on responses. Total 27 runs were suggested by the software. Factors and responses considered for study were shown in table 1. Ranges considered were based upon previous univariate chromatographic separation studies. For amount of organic range was 60 to $80 \% \mathrm{v} / \mathrm{v}$, for $\mathrm{pH}$ of buffer 3 to 5 and for flow rate 0.8 to $1.2 \mathrm{~mL} \mathrm{~min}^{-1} .7,8$.

\subsection{Optimization}

\subsubsection{Screening design for suitable chromatographic condition:}

Determination of suitable column and solvent system based on peak parameters. Methanol: water/ methanol: buffer (Amonium formate)/ ACN: water and ACN: buffer, these four mobile phases were selected for screening study on C8 and 
C18 columns at pH 3.0, 4.0 and 5.0. These mobile phases were screened by varying the organic phase composition from 60 to $80 \% \mathrm{v} / \mathrm{v}$ and flow rate from 0.8 to $1.2 \mathrm{~mL} / \mathrm{min}$ flow rate consider to be standard $1 \mathrm{ml} / \mathrm{min}$ because result are not satisfactory with 0.8 and $1.2 \mathrm{ml} / \mathrm{min}$.
3.2.2 Results of various trials, having organic phase composition $60 \% \mathrm{v} / \mathrm{v}$ are shown in following tables.

Table 1: Trials performed on C8 column at mobile phase $(60: 40 \mathrm{v} / \mathrm{v})$ with aqueous phase $\mathrm{pH} 3$

\begin{tabular}{|l|l|l|l|}
\hline Sr.no. & Composition & Observation & Remarks \\
\hline 1 & $\begin{array}{l}\text { Methanol: buffer } \\
(60: 40 \mathrm{v} / \mathrm{v})\end{array}$ & No peak appearance & Not satisfactory \\
\hline 2 & $\begin{array}{l}\text { Methanol: water } \\
(60: 40 \mathrm{v} / \mathrm{v})\end{array}$ & No peak appearance & Not satisfactory \\
\hline 3 & $\begin{array}{l}\text { ACN: buffer } \\
(60: 40 \mathrm{v} / \mathrm{v})\end{array}$ & $\begin{array}{l}\text { Greater peak asymmetry and lower } \\
\text { theoretical plates }\end{array}$ & Not satisfactory \\
\hline 4 & $\begin{array}{l}\text { ACN: water } \\
(60: 40 \mathrm{v} / \mathrm{v})\end{array}$ & $\begin{array}{l}\text { Greater peak asymmetry and lower } \\
\text { theoretical plates }\end{array}$ & Not satisfactory \\
\hline
\end{tabular}

Table 2: Trials performed on C18 column at mobile phase $(60: 40 \mathrm{v} / \mathrm{v})$ with aqueous phase pH 3

\begin{tabular}{|l|l|l|l|}
\hline Sr. no. & Composition & Observation & Remarks \\
\hline 1 & $\begin{array}{l}\text { Methanol: buffer } \\
(60: 40 \mathrm{v} / \mathrm{v})\end{array}$ & $\begin{array}{l}\text { Greater peak Asymmetry and lower } \\
\text { theoretical plates }\end{array}$ & Not satisfactory \\
\hline 2 & $\begin{array}{l}\text { Methanol: water } \\
(60: 40 \mathrm{v} / \mathrm{v})\end{array}$ & No peak appearance & Not satisfactory \\
\hline 3 & $\begin{array}{l}\text { ACN: buffer } \\
(60: 40 \mathrm{v} / \mathrm{v})\end{array}$ & $\begin{array}{l}\text { Less peak asymmetry but less } \\
\text { theoretical plates }\end{array}$ & Not satisfactory \\
\hline 4 & $\begin{array}{l}\text { ACN: water } \\
(60: 40 \mathrm{v} / \mathrm{v})\end{array}$ & $\begin{array}{l}\text { Less peak asymmetry with more } \\
\text { theoretical plates }\end{array}$ & Satisfactory \\
\hline
\end{tabular}

Table 3: Trials performed on C8 column at mobile phase $(60: 40 \mathrm{v} / \mathrm{v})$ with aqueous phase pH 4

\begin{tabular}{|l|l|l|l|}
\hline Sr.no. & Composition & Observation & Remarks \\
\hline 1 & $\begin{array}{l}\text { Methanol: buffer } \\
(60: 40 \mathrm{v} / \mathrm{v})\end{array}$ & No peak appearance & Not satisfactory \\
\hline 2 & $\begin{array}{l}\text { Methanol: water } \\
(60: 40 \mathrm{v} / \mathrm{v})\end{array}$ & No peak appearance & Not satisfactory \\
\hline 3 & $\begin{array}{l}\text { ACN: buffer } \\
(60: 40 \mathrm{v} / \mathrm{v})\end{array}$ & More retention time & Not satisfactory \\
\hline 4 & $\begin{array}{l}\text { ACN: } \text { water } \\
(60: 40 \mathrm{v} / \mathrm{v})\end{array}$ & More retention time & Not satisfactory \\
\hline
\end{tabular}

Table 4: Trials performed on C18 column at mobile phase $(60: 40 \mathrm{v} / \mathrm{v})$ with aqueous phase pH 4

\begin{tabular}{|c|c|c|c|}
\hline Sr.no. & Composition & Observation & Remarks \\
\hline 1 & $\begin{array}{l}\text { Methanol: buffer } \\
(60: 40 \mathrm{v} / \mathrm{v})\end{array}$ & No peak appearance & Not satisfactory \\
\hline 2 & $\begin{array}{l}\text { Methanol: water } \\
(60: 40 \mathrm{v} / \mathrm{v})\end{array}$ & No peak appearance & Not satisfactory \\
\hline 3 & $\begin{array}{l}\text { ACN: buffer } \\
(60: 40 \mathrm{v} / \mathrm{v})\end{array}$ & Good peak properties & Satisfactory \\
\hline 4 & $\begin{array}{l}\text { ACN: water } \\
(60: 40 \mathrm{v} / \mathrm{v})\end{array}$ & More retention time & Not satisfactory \\
\hline
\end{tabular}

Table 5: Trials performed on C8 column at mobile phase $(60: 40 \mathrm{v} / \mathrm{v})$ with aqueous phase pH 5

\begin{tabular}{|l|l|l|l|}
\hline Sr. no. & Composition & Observation & Remarks \\
\hline 1 & $\begin{array}{l}\text { Methanol: buffer } \\
(60: 40 \mathrm{v} / \mathrm{v})\end{array}$ & No peak observed & Not satisfactory \\
\hline 2 & $\begin{array}{l}\text { Methanol: water } \\
(60: 40 \mathrm{v} / \mathrm{v})\end{array}$ & No peak appearance & Not satisfactory \\
\hline 3 & $\begin{array}{l}\text { ACN: buffer } \\
(60: 40 \mathrm{v} / \mathrm{v})\end{array}$ & $\begin{array}{l}\text { Greater peak asymmetry with less } \\
\text { theoretical plates }\end{array}$ & Not satisfactory \\
\hline 4 & $\begin{array}{l}\text { ACN: } \text { water } \\
(60: 40 \mathrm{v} / \mathrm{v})\end{array}$ & More retention time & Not satisfactory \\
\hline
\end{tabular}


Table 6: Trials performed on C18 column at mobile phase $(60: 40 \mathrm{v} / \mathrm{v})$ with aqueous phase pH 5

\begin{tabular}{|c|c|c|c|}
\hline Sr. no. & Composition & Observation & Remarks \\
\hline 1 & $\begin{array}{l}\text { Methanol: } \\
\text { buffer }(60: 40 \mathrm{v} / \mathrm{v})\end{array}$ & No peak observed & Not satisfactory \\
\hline 2 & $\begin{array}{l}\text { Methanol: water } \\
(60: 40 \mathrm{v} / \mathrm{v})\end{array}$ & No peak observed & Not satisfactory \\
\hline 3 & $\begin{array}{l}\text { ACN: buffer } \quad(60: 40 \\
\mathrm{v} / \mathrm{v})\end{array}$ & More retention time & Not satisfactory \\
\hline 4 & $\begin{array}{l}\text { ACN: water } \\
(60: 40 \mathrm{v} / \mathrm{v})\end{array}$ & More retention time & Not satisfactory \\
\hline
\end{tabular}

3.2.3 Results of various trials, having organic phase composition $70 \% \mathrm{v} / \mathrm{v}$ are shown in following tables.

Table 7: Trials performed on C8 column at mobile phase $(70: 30 \mathrm{v} / \mathrm{v})$ with aqueous phase $\mathrm{pH} 3$

\begin{tabular}{|c|c|c|c|}
\hline $\begin{array}{l}\text { Sr. } \\
\text { no. }\end{array}$ & Composition & Observation & Remarks \\
\hline 1 & $\begin{array}{l}\text { Methanol: buffer } \\
(70: 30 \mathrm{v} / \mathrm{v})\end{array}$ & $\begin{array}{l}\text { Greater peak asymmetry and lower } \\
\text { theoretical plates }\end{array}$ & Not satisfactory \\
\hline 2 & $\begin{array}{l}\text { Methanol: water } \\
(70: 30 \mathrm{v} / \mathrm{v})\end{array}$ & More retention time & Not satisfactory \\
\hline 3 & $\begin{array}{l}\text { ACN: buffer } \\
(70: 30 \mathrm{v} / \mathrm{v})\end{array}$ & $\begin{array}{l}\text { Greater peak asymmetry and lower } \\
\text { theoretical plates }\end{array}$ & Not satisfactory \\
\hline 4 & $\begin{array}{l}\text { ACN: water } \\
(70: 30 \mathrm{v} / \mathrm{v})\end{array}$ & Greater peak asymmetry & Not satisfactory \\
\hline
\end{tabular}

Table 8: Trials performed on C18 column at mobile phase $(70: 30 \mathrm{v} / \mathrm{v})$ with aqueous phase pH 3

\begin{tabular}{|l|l|l|l|}
\hline $\begin{array}{l}\text { Sr. } \\
\text { no. }\end{array}$ & Composition & Observation & Remarks \\
\hline 1 & $\begin{array}{l}\text { Methanol: buffer } \\
(70: 30 \mathrm{v} / \mathrm{v})\end{array}$ & More retention time & Not satisfactory \\
\hline 2 & $\begin{array}{l}\text { Methanol: water } \\
(70: 30 \mathrm{v} / \mathrm{v})\end{array}$ & More retention time & Not satisfactory \\
\hline 3 & $\begin{array}{l}\text { ACN: buffer } \\
(70: 30 \mathrm{v} / \mathrm{v})\end{array}$ & $\begin{array}{l}\text { Less peak asymmetry with more theoretical } \\
\text { plates and good retention time }\end{array}$ & More satisfactory \\
\hline 4 & $\begin{array}{l}\text { ACN: water } \\
(70: 30 \mathrm{v} / \mathrm{v})\end{array}$ & $\begin{array}{l}\text { Less peak asymmetry with more theoretical } \\
\text { plates and good retention time }\end{array}$ & satisfactory \\
\hline
\end{tabular}

Table 9: Trials performed on C8 column at mobile phase $(70: 30 \mathrm{v} / \mathrm{v})$ with aqueous phase pH 4

\begin{tabular}{|l|l|l|l|}
\hline Sr.no. & Composition & Observation & Remarks \\
\hline 1 & $\begin{array}{l}\text { Methanol: buffer } \\
(70: 30 \mathrm{v} / \mathrm{v})\end{array}$ & $\begin{array}{l}\text { Greater peak asymmetry with lower } \\
\text { theoretical plates }\end{array}$ & $\begin{array}{l}\text { Not } \\
\text { satisfactory }\end{array}$ \\
\hline 2 & $\begin{array}{l}\text { Methanol: water } \\
(70: 30 \mathrm{v} / \mathrm{v})\end{array}$ & No peak appearance & $\begin{array}{l}\text { Not } \\
\text { satisfactory }\end{array}$ \\
\hline 3 & $\begin{array}{l}\text { ACN: buffer } \\
(70: 30 \mathrm{v} / \mathrm{v})\end{array}$ & $\begin{array}{l}\text { Less peak asymmetry with more theoretical } \\
\text { plates and good retention time }\end{array}$ & satisfactory \\
\hline 4 & $\begin{array}{l}\text { ACN: } \text { water } \\
(70: 30 \mathrm{v} / \mathrm{v})\end{array}$ & $\begin{array}{l}\text { Greater peak asymmetry with more } \\
\text { theoretical plates }\end{array}$ & $\begin{array}{l}\text { Not } \\
\text { satisfactory }\end{array}$ \\
\hline
\end{tabular}

Table 10: Trials performed on C18 column at mobile phase (70:30 v/v) with aqueous phase pH 4

\begin{tabular}{|c|c|c|c|}
\hline Sr.no. & Composition & Observation & Remarks \\
\hline 1 & $\begin{array}{l}\text { Methanol: buffer } \\
(70: 30 \mathrm{v} / \mathrm{v})\end{array}$ & More retention time & Not satisfactory \\
\hline 2 & $\begin{array}{l}\text { Methanol: water } \\
(70: 30 \mathrm{v} / \mathrm{v})\end{array}$ & More retention time & Not satisfactory \\
\hline 3 & $\begin{array}{l}\text { ACN: buffer } \\
(70: 30 \mathrm{v} / \mathrm{v})\end{array}$ & $\begin{array}{l}\text { Less peak asymmetry with more } \\
\text { theoretical plates and good retention time }\end{array}$ & Satisfactory \\
\hline 4 & $\begin{array}{l}\text { ACN: water } \\
(70: 30 \mathrm{v} / \mathrm{v})\end{array}$ & $\begin{array}{l}\text { Less peak asymmetry with more } \\
\text { theoretical plates and good retention time }\end{array}$ & Satisfactory \\
\hline
\end{tabular}


Table 11: Trials performed on C8 column at mobile phase $(70: 30 \mathrm{v} / \mathrm{v})$ with aqueous phase pH 5

\begin{tabular}{|l|l|l|l|}
\hline Sr. no. & Composition & Observation & Remarks \\
\hline 1 & $\begin{array}{l}\text { Methanol: buffer } \\
(70: 30 \mathrm{v} / \mathrm{v})\end{array}$ & More retention time & Not satisfactory \\
\hline 2 & $\begin{array}{l}\text { Methanol: water } \\
(70: 30 \mathrm{v} / \mathrm{v})\end{array}$ & Greater peak asymmetry & Not satisfactory \\
\hline 3 & $\begin{array}{l}\text { ACN: buffer } \\
(70: 30 \mathrm{v} / \mathrm{v})\end{array}$ & $\begin{array}{l}\text { Greater peak asymmetry with less } \\
\text { theoretical plates }\end{array}$ & Not satisfactory \\
\hline 4 & $\begin{array}{l}\text { ACN: water } \\
(70: 30 \mathrm{v} / \mathrm{v})\end{array}$ & $\begin{array}{l}\text { Greater peak asymmetry with less } \\
\text { theoretical plates }\end{array}$ & Not satisfactory \\
\hline
\end{tabular}

Table 12: Trials performed on C18 column at mobile phase $(70: 30 \mathrm{v} / \mathrm{v})$ with aqueous phase pH 5

\begin{tabular}{|l|l|l|l|}
\hline Sr. no. & Composition & Observation & Remarks \\
\hline 1 & Methanol: buffer $(70: 30 \mathrm{v} / \mathrm{v})$ & More retention time & Not satisfactory \\
\hline 2 & Methanol: water $(70: 30 \mathrm{v} / \mathrm{v})$ & More retention time & Not satisfactory \\
\hline 3 & $\begin{array}{l}\text { ACN: buffer } \\
(70: 30 \mathrm{v} / \mathrm{v})\end{array}$ & $\begin{array}{l}\text { Less peak asymmetry with more theoretical } \\
\text { plates and good retention time }\end{array}$ & More satisfactory \\
\hline 4 & $\begin{array}{l}\text { ACN: water } \\
(70: 30 \mathrm{v} / \mathrm{v})\end{array}$ & $\begin{array}{l}\text { pess peak asymmetry with more theoretical } \\
\text { plates and good retention time }\end{array}$ & satisfactory \\
\hline
\end{tabular}

3.2.4 Results of various trials, having organic phase composition $80 \% \mathrm{v} / \mathrm{v}$ are shown in following tables.

Table 13: Trials performed on $\mathrm{C} 8$ column at mobile phase $(80: 20 \mathrm{v} / \mathrm{v})$ with aqueous phase $\mathrm{pH} 3$

\begin{tabular}{|c|c|c|c|}
\hline Sr. no. & Composition & Observation & Remarks \\
\hline 1 & $\begin{array}{l}\text { Methanol: buffer } \\
(80: 20 \mathrm{v} / \mathrm{v})\end{array}$ & $\begin{array}{l}\text { Greater peak asymmetry and } \\
\text { More retention time }\end{array}$ & Not satisfactory \\
\hline 2 & $\begin{array}{l}\text { Methanol: water } \\
(80: 20 \mathrm{v} / \mathrm{v})\end{array}$ & Greater peak asymmetry & Not satisfactory \\
\hline 3 & $\begin{array}{l}\text { ACN: buffer } \\
(80: 20 \mathrm{v} / \mathrm{v})\end{array}$ & Greater peak asymmetry & Not satisfactory \\
\hline 4 & $\begin{array}{l}\text { ACN: water } \\
(80: 20 \mathrm{v} / \mathrm{v})\end{array}$ & $\begin{array}{l}\text { Greater peak asymmetry and } \\
\text { lower theoretical plates }\end{array}$ & Not satisfactory \\
\hline
\end{tabular}

Table 14: Trials performed on C18 column at mobile phase $(80: 20 \mathrm{v} / \mathrm{v})$ with aqueous phase pH 3

\begin{tabular}{|l|l|l|l|}
\hline Sr. no. & Composition & Observation & Remarks \\
\hline 1 & Methanol: buffer $(80: 20 \mathrm{v} / \mathrm{v})$ & Lower theoretical plates & Not satisfactory \\
\hline 2 & Methanol: water $(80: 20 \mathrm{v} / \mathrm{v})$ & Lower theoretical plates & Not satisfactory \\
\hline 3 & ACN: buffer $(80: 20 \mathrm{v} / \mathrm{v})$ & Less retention time & Not satisfactory \\
\hline 4 & ACN: water $(80: 20 \mathrm{v} / \mathrm{v})$ & Lower theoretical plates & satisfactory \\
\hline
\end{tabular}

Table 15: Trials performed on C8 column at mobile phase $(80: 20 \mathrm{v} / \mathrm{v})$ with aqueous phase pH 4

\begin{tabular}{|l|l|l|l|}
\hline Sr.no. & Composition & Observation & Remarks \\
\hline 1 & $\begin{array}{l}\text { Methanol: buffer } \\
(80: 20 \mathrm{v} / \mathrm{v})\end{array}$ & $\begin{array}{l}\text { Greater peak asymmetry and lower } \\
\text { theoretical plates with more retention } \\
\text { time }\end{array}$ & Not satisfactory \\
\hline 2 & $\begin{array}{l}\text { Methanol: water } \\
(80: 20 \mathrm{v} / \mathrm{v})\end{array}$ & Greater peak asymmetry & Not satisfactory \\
\hline 3 & $\begin{array}{l}\text { ACN: buffer } \\
(80: 20 \mathrm{v} / \mathrm{v})\end{array}$ & $\begin{array}{l}\text { Greater peak asymmetry and lower } \\
\text { theoretical plates }\end{array}$ & Not satisfactory \\
\hline 4 & $\begin{array}{l}\text { ACN: water } \\
(80: 20 \mathrm{v} / \mathrm{v})\end{array}$ & $\begin{array}{l}\text { Greater peak asymmetry and lower } \\
\text { theoretical plates }\end{array}$ & Not satisfactory \\
\hline
\end{tabular}

Table 16: Trials performed on C18 column at mobile phase $(80: 20 \mathrm{v} / \mathrm{v})$ with aqueous phase pH 4

\begin{tabular}{|l|l|l|l|}
\hline Sr.no. & Composition & Observation & Remarks \\
\hline 1 & $\begin{array}{l}\text { Methanol: buffer } \\
(80: 20 \mathrm{v} / \mathrm{v})\end{array}$ & No peak appearance & Not satisfactory \\
\hline 2 & $\begin{array}{l}\text { Methanol: water } \\
(80: 20 \mathrm{v} / \mathrm{v})\end{array}$ & No peak appearance & Not satisfactory \\
\hline 3 & $\begin{array}{l}\text { ACN: buffer } \\
(80: 20 \mathrm{v} / \mathrm{v})\end{array}$ & Less retention time & Satisfactory \\
\hline 4 & $\begin{array}{l}\text { ACN: water } \\
(80: 20 \mathrm{v} / \mathrm{v})\end{array}$ & Good peak properties & satisfactory \\
\hline
\end{tabular}


Table 17: Trials performed on $\mathrm{C8}$ column at mobile phase $(80: 20 \mathrm{v} / \mathrm{v})$ with aqueous phase pH 5

\begin{tabular}{|c|c|c|c|}
\hline Sr. no. & Composition & Observation & Remarks \\
\hline 1 & $\begin{array}{l}\text { Methanol: buffer } \\
(80: 20 \mathrm{v} / \mathrm{v})\end{array}$ & Greater peak asymmetry & Not satisfactory \\
\hline 2 & $\begin{array}{l}\text { Methanol: water } \\
(80: 20 \mathrm{v} / \mathrm{v})\end{array}$ & less theoretical plates & Not satisfactory \\
\hline 3 & $\begin{array}{l}\text { ACN: buffer } \\
(80: 20 \mathrm{v} / \mathrm{v})\end{array}$ & $\begin{array}{l}\text { Greater peak asymmetry with less } \\
\text { theoretical plates }\end{array}$ & Not satisfactory \\
\hline 4 & $\begin{array}{l}\text { ACN: water } \\
(80: 20 \mathrm{v} / \mathrm{v})\end{array}$ & $\begin{array}{l}\text { Greater peak asymmetry with less } \\
\text { theoretical plates }\end{array}$ & Not satisfactory \\
\hline
\end{tabular}

Table 18: Trials performed on C18 column at mobile phase $(80: 20 \mathrm{v} / \mathrm{v})$ with aqueous phase pH 5

\begin{tabular}{|l|l|l|l|}
\hline Sr. no. & Composition & Observation & Remarks \\
\hline 1 & $\begin{array}{l}\text { Methanol: buffer } \\
(80: 20 \mathrm{v} / \mathrm{v})\end{array}$ & lower theoretical plates & Not satisfactory \\
\hline 2 & $\begin{array}{l}\text { Methanol: water } \\
(80: 20 \mathrm{v} / \mathrm{v})\end{array}$ & lower theoretical plates & Not satisfactory \\
\hline 3 & $\begin{array}{l}\text { ACN: buffer } \\
(80: 20 \mathrm{v} / \mathrm{v})\end{array}$ & Good peak properties & satisfactory \\
\hline 4 & $\begin{array}{l}\text { ACN: water } \\
(80: 20 \mathrm{v} / \mathrm{v})\end{array}$ & Lower retention time & Not satisfactory \\
\hline
\end{tabular}

3.3 Optimized trials suggested by software based on desirability value

This methodology is initially based on constructing a desirability function for each individual response. The scale of individual desirability function ranges between $\mathrm{i}=0$, for completely undesirable response and $\mathrm{i}=1$, for fully desired response. Selection of trial was based on maximum desirability value. Therefore, first trial which was having desirability one (i=1) selected for method optimization..$^{8,9,10 .}$

Table 19: Optimized trials suggested by software based on desirability value

\begin{tabular}{|l|l|l|l|l|l|l|l|}
\hline Sr. no. & $\begin{array}{l}\text { Amount } \\
\text { of CAN }\end{array}$ & $\begin{array}{l}\text { pH of } \\
\text { buffer }\end{array}$ & $\begin{array}{l}\text { Flow } \\
\text { rate }\end{array}$ & $\begin{array}{l}\text { Retention } \\
\text { time }\end{array}$ & $\begin{array}{l}\text { Tailing } \\
\text { factor }\end{array}$ & $\begin{array}{l}\text { Theoretical } \\
\text { plates }\end{array}$ & Desirability \\
\hline $\mathbf{1}$ & $\mathbf{7 2 . 0 3}$ & $\mathbf{5 . 0}$ & $\mathbf{1 . 0}$ & $\mathbf{5 . 6}$ & $\mathbf{0 . 9 6}$ & 9084.48 & $\mathbf{1 . 0}$ \\
\hline 2 & 72.00 & 5.0 & 1.0 & 5.9 & 1.01 & 9124.84 & 0.9 \\
\hline 3 & 72.56 & 5.0 & 1.1 & 5.7 & 1.03 & 9025.81 & 1.0 \\
\hline 4 & 70.02 & 5.0 & 1.0 & 5.76 & 0.99 & 8997.3 & 0.96 \\
\hline
\end{tabular}

\subsubsection{Optimized chromatographic conditions}

Mobile phase: Ammonium format buffer: ACN (28:72 v/v), pH of buffer: 5, Analytical column: $\mathrm{C}_{18}$ column Waters XBridge $(4.6 \times 250 \mathrm{~mm}$ id. particle size $5 \mu \mathrm{m})$, UV detection: $247 \mathrm{~nm}$, Injection volume: $20 \mu \mathrm{L}$, Flow rate: $1.00 \mathrm{~mL}^{\mathrm{min}}{ }^{-1}$, Temperature: Ambient, Run time: $10 \mathrm{~min}$

\subsection{Effect of independent variables}

\subsubsection{Effect of independent variables on retention time (Y):}

The equation for response surface quadratic model is as follows

$\mathrm{Y}_{1}=+5.56-2.58 \mathrm{X}_{1}+0.50 \mathrm{X}_{2}-1.52 \mathrm{X}_{3}+0.14 \mathrm{X}_{1} \mathrm{X}_{2}+0.79 \mathrm{X}_{1} \mathrm{X}_{3}+-$ $0.099 \mathrm{X}_{2} \mathrm{X}_{3}+1.45 \mathrm{X}_{1}^{2}+0.46 \mathrm{X}_{2}{ }^{2}+0.96 \mathrm{X}_{3}{ }^{2}$ (1)

Where, $\mathrm{X}_{1}=\mathrm{A}, \mathrm{X}_{2}=\mathrm{B}$ and $\mathrm{X}_{3}=\mathrm{C}$

a graphical representation of amount of ACN (A) and flow rate $(\mathrm{C})$, while $\mathrm{pH}$ of buffer $(\mathrm{B})$ is maintained constant at its optimum of 5 . An increase in flow rate resulted in decrease in retention time $\left(\mathrm{Y}_{1}\right)$, while increase in $\% \mathrm{v} / \mathrm{v}$ of buffer (or amount of buffer) resulted in increase in retention time ( $\left.\mathrm{Y}_{1}\right)$. Combination of amount of buffer and flow rate showed decrease in response.

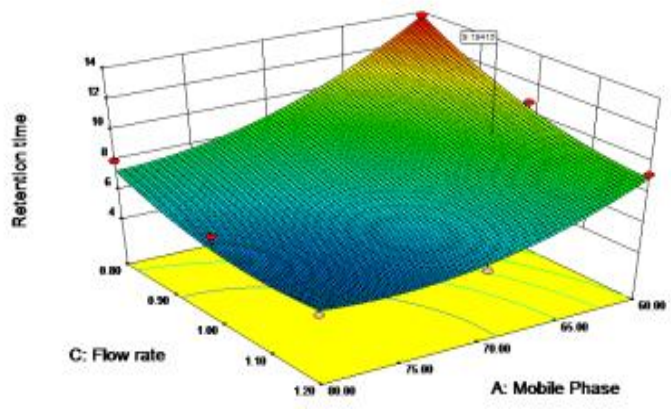

Figure 2(a): Three-dimensional plot for retention time as a function of flow rate and amount of ACN. Constant factor (pH of buffer- 5.0)

Fig.3 (b) shows a graphical representation of $\mathrm{pH}$ of buffer (B) and amount of buffer $(\mathrm{A})$, while flow rate $(\mathrm{C})$ is maintained constant at its optimum of $1 \mathrm{~mL} \mathrm{~min}^{-1}$. Increase in $\mathrm{pH}$ of buffer showed increase in retention time $\left(\mathrm{Y}_{1}\right)$, also increase in amount of buffer showed synergistic effect on $Y_{1}$. Combination of amount of buffer and $\mathrm{pH}$ of buffer showed antagonistic effect on response. 


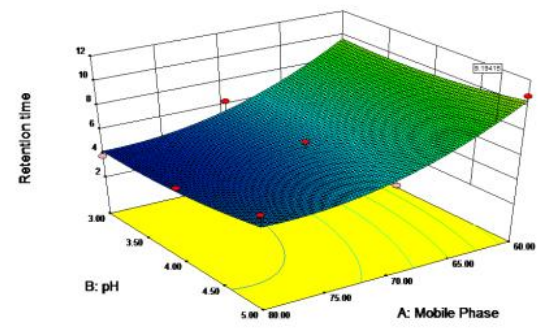

Figure 3(b): Three-dimensional plot for retention time as a function of $\mathrm{pH}$ of buffer and amount of buffer.

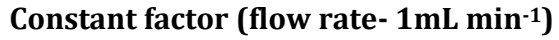

Fig.4.(c) shows a graphical representation of $\mathrm{pH}$ of buffer (B) and flow rate $(\mathrm{C})$, while amount of buffer $(\mathrm{A})$ is maintained constant at its optimum of $40 \% \mathrm{v} / \mathrm{v}$. An increase in flow rate showed antagonistic effect on response $\left(\mathrm{Y}_{1}\right)$, while increase in $\mathrm{pH}$ of buffer showed synergistic effect on the response. $\mathrm{pH}$ of buffer and flow rate combination found for having synergistic effect on response.

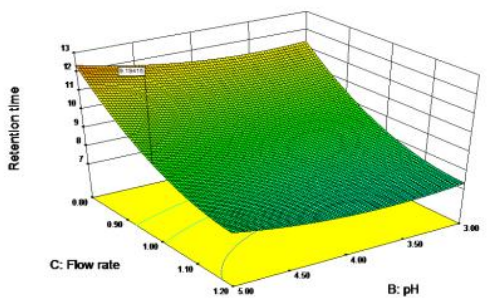

Figure 4(c): Three-dimensional plot for retention time as a function of flow rate and $\mathrm{pH}$ of buffer. Constant factor (\% $\mathrm{v} / \mathrm{v}$ of buffer- 40$)$

Fit summary: Quadratic model was suggested by the software.

ANOVA: ANOVA of developed Full three level factorial model for retention time $\left(\mathrm{Y}_{1}\right)$.

Values of "Prob > F" (p- value) less than 0.0500 indicate model terms are significant.

In this case $\mathrm{A}, \mathrm{B}, \mathrm{BC}, \mathrm{A}^{2}$ and $\mathrm{C}^{2}$ are significant model terms.

Table 20: Significance of $\boldsymbol{p}$ value on model terms of retention time

\begin{tabular}{|l|l|l|l|}
\hline Model terms & $\boldsymbol{p}$ value & Effect of factor & Remarks \\
\hline $\mathrm{A}\left(\mathrm{X}_{1}\right)$ & $\mathbf{0 . 0 0 0 1}$ & -2.58 & Significant \\
\hline $\mathrm{B}\left(\mathrm{X}_{2}\right)$ & $\mathbf{0 . 0 1 1 5}$ & +0.50 & Significant \\
\hline $\mathrm{C}\left(\mathrm{X}_{3}\right)$ & $\mathbf{0 . 0 0 0 1}$ & -1.52 & Significant \\
\hline $\mathrm{AB}\left(\mathrm{X}_{1} \mathrm{X}_{2}\right)$ & 0.5381 & +0.14 & Insignificant \\
\hline $\mathrm{AC}\left(\mathrm{X}_{1} \mathrm{X}_{3}\right)$ & $\mathbf{0 . 0 0 2 1}$ & +0.79 & Significant \\
\hline $\mathrm{BC}\left(\mathrm{X}_{2} \mathrm{X}_{3}\right)$ & 0.6555 & -0.099 & Insignificant \\
\hline $\mathrm{A}^{2}\left(\mathrm{X}^{2}\right)$ & $\mathbf{0 . 0 0 0 2}$ & +1.45 & Significant \\
\hline $\mathrm{B}^{2}\left(\mathrm{X}^{2}\right)$ & 0.1565 & +0.46 & Insignificant \\
\hline $\mathrm{C}^{2}\left(\mathrm{X}^{2}\right)$ & $\mathbf{0 . 0 0 6 1}$ & +0.96 & Significant \\
\hline Overall model & $\mathbf{0 . 0 0 2 9}$ & - & Significant \\
\hline
\end{tabular}

\subsubsection{Effect of independent variables on tailing factor} $\left(\mathrm{Y}_{2}\right)$ :

After applying experimental design, suggested Response Surface Linear Model was found to be significant with model $\mathrm{F}$ value of $23.79, p$ value less than 0.005 and $\mathrm{R}^{2}$ value of 0.7563 . There is only a $0.01 \%$ chance that a "Model FValue" this large could occur due to noise. Values of \% C.V. and adjusted $\mathrm{R}^{2}$ were 3.78 and 0.7245 respectively. The model for response $\mathrm{Y}_{2}$ (tailing factor) is as follows:

$$
\mathrm{Y}_{2}=\quad+0.98+0.071 \mathrm{X}_{1}-0.021 \mathrm{X}_{2}+3.333 \mathrm{E}-003 \mathrm{X}_{3}
$$

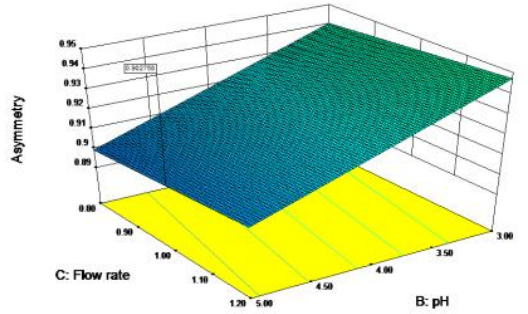

Figure 5(a): Three-dimentional plot for tailing factor as a function of flow rate and amount of buffer. Constant factor (pH of buffer- 5)

Fig.6.(b) shows a graphical representation of $\mathrm{pH}$ of buffer (B) and amount of buffer (A), while flow rate (C) is maintained constant at its optimum of $1.0 \mathrm{~mL} \mathrm{~min}^{-1}$. An increase in $\mathrm{pH}$ of buffer had antagonistic effect on response $\left(\mathrm{Y}_{2}\right)$ while increase in amount of ACN showed synergistic effect on it. Amount and pH of buffer combination showed antagonistic effect on response.

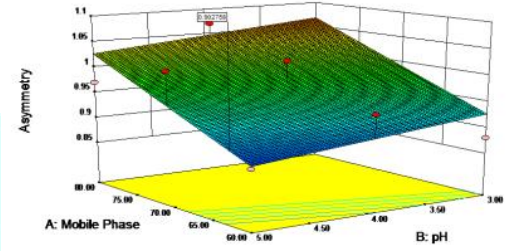

Figure 6(b): Three-dimentional plot for tailing factor as a function of $\mathrm{pH}$ of buffer and $\% \mathrm{v} / \mathrm{v}$ of buffer. Constant factor (flow rate- $1 \mathrm{~mL} \mathrm{~min}^{-1}$ )

Fig.7.(c) shows a graphical representation of $\mathrm{pH}$ of buffer (B) and flow rate (C), while amount of buffer (A) is maintained constant at its optimum $40 \% \mathrm{v} / \mathrm{v}$. An increase in $\mathrm{pH}$ of buffer and flow rate showed antagonistic effect on response $\left(\mathrm{Y}_{2}\right)$. Combination of flow rate and $\mathrm{pH}$ of buffer showed synergistic effect.

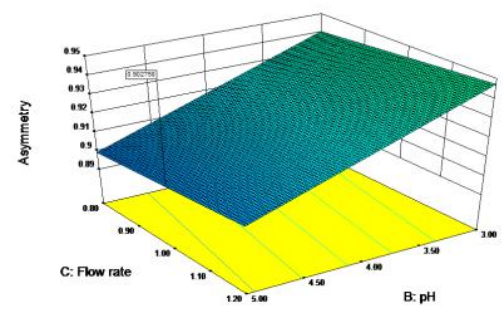

Figure 7(c): Three-dimentional plot for tailing factor as a function of flow rate and $\mathrm{pH}$ of buffer. Constant factor (amount of buffer) 
Fit summary: Response Surface Linear Model was suggested by the software.

ANOVA: ANOVA of developed CCD model for tailing factor $\left(Y_{2}\right)$.
Values of "Prob > F" (p- value) less than 0.0500 indicate model terms are significant.

In this case A, B are significant model terms.

Table 21: Significance of $\boldsymbol{p}$ value on model terms of tailing factor

\begin{tabular}{|l|l|l|l|}
\hline Model terms & $\boldsymbol{p}$ value & Effect of factor & Remarks \\
\hline $\mathrm{A}\left(\mathrm{X}_{1}\right)$ & $\mathbf{0 . 0 9 0}$ & +0.071 & Significant \\
\hline $\mathrm{B}\left(\mathrm{X}_{2}\right)$ & $\mathbf{0 . 0 2 7 1}$ & -0.021 & Significant \\
\hline $\mathrm{C}\left(\mathrm{X}_{3}\right)$ & 0.7054 & +3.333 & Insignificant \\
\hline Overall model & 0.044 & - & Significant \\
\hline
\end{tabular}

For response $Y_{2}$, factor $X_{1}$ and $X_{3}$ was having synergistic effect with $p$ value 0.090 and 0.7054 . Therefore we can conclude that increment in amount of ACN and flow rate was responsible for decrease in tailing factor and thus showed the direct relationship between them. Terms $\mathrm{X}_{2}$ were responsible for significant increase in tailing factor with $p$ values -0.0271 . Therefore we can conclude that combination of factors showed inverse relationship with response. Factors amount of ACN and combination of flow rate and amount of buffer differs from zero with a great margin therefore they were having more significant effect than other factors.

\subsubsection{Effect of independent variables on theoretical} plates $\left(\mathrm{Y}_{3}\right)$ :

After applying experimental design, suggested Response Surface Quadratic Model was found to be significant with model $\mathrm{F}$ value of $4.38, p$ value less than 0.005 and $\mathrm{R}^{2}$ value of 0.6988 . There is only a $0.43 \%$ chance that a "Model FValue" this large could occur due to noise. Values of \% C.V. and adjusted $\mathrm{R}^{2}$ were 18.43 and 0.5394 respectively. The model for response $\mathrm{Y}_{3}$ (theoretical plates) is as follows:

$\mathrm{Y}_{3}=+6409.99-1700.54 \mathrm{X}_{1}+283.43 \mathrm{X}_{2}-322.85 \mathrm{X}_{3}-602.20 \mathrm{X}_{1} \mathrm{X}_{2}+$ $445.98 \mathrm{X}_{1} \mathrm{X}_{3}-323.61 \mathrm{X}_{2} \mathrm{X}_{3}+243.15 \mathrm{X}_{1}+510.74 \mathrm{X}_{2}{ }_{2}+2176.65 \mathrm{X}_{3}$ (3)

Fig.8 (a) shows a graphical representation of amount of ACN (A) and flow rate (C), while pH of buffer (B) is varying from 3-5.

An increase in flow rate and amount of ACN showed antagonistic effect on response $\left(\mathrm{Y}_{3}\right)$ individually, while their combination showed synergistic effect on response. 1,10 .

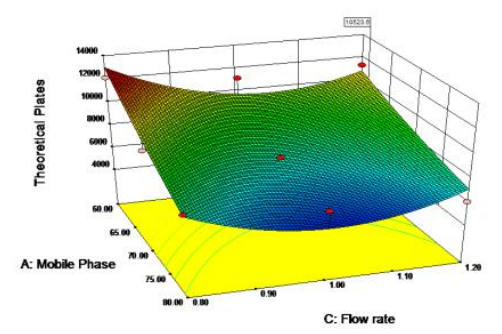

Figure 8(a): Three-dimensional plot for theoretical plates as a function of flow rate and amount of ACN. Constant factor pH-5

Fig.9.(b) shows a graphical representation of amount of $\mathrm{ACN}(\mathrm{A})$ and $\mathrm{pH}$ of buffer (B), while flow rate (C) is maintained constant at its optimum value $1 \mathrm{~mL} \mathrm{~min}-1$. An increase in $\mathrm{pH}$ of buffer showed increase in number of theoretical plates $\left(\mathrm{Y}_{3}\right)$, while increase in amount of ACN showed antagonistic effect on response. Combination of amount of buffer and $\mathrm{pH}$ of buffer showed antagonistic effect on it.

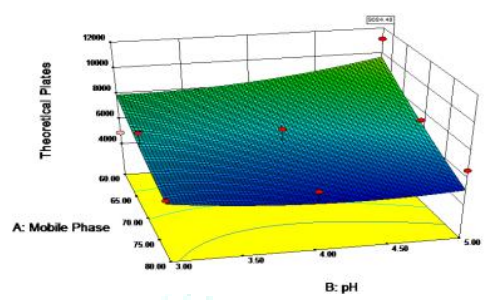

Figure 9(b): Three-dimensional plot for theoretical plates as a function of $\mathrm{pH}$ of buffer and $\% \mathrm{v} / \mathrm{v}$ of buffer. Constant factor (flow rate- $1 \mathrm{~mL} \mathrm{~min}^{-1}$ )

Fig.10.(c) shows a graphical representation of flow rate (C) and $\mathrm{pH}$ of buffer (B), while amount of ACN (A) is maintained constant at its optimum value $70 \% \mathrm{v} / \mathrm{v}$. An increase in $\mathrm{pH}$ of buffer showed increase in number of theoretical plates $\left(\mathrm{Y}_{3}\right)$, while, increase in flow rate showed antagonistic effect on response. Combination of flow rate and $\mathrm{pH}$ of buffer showed antagonistic effect on response.

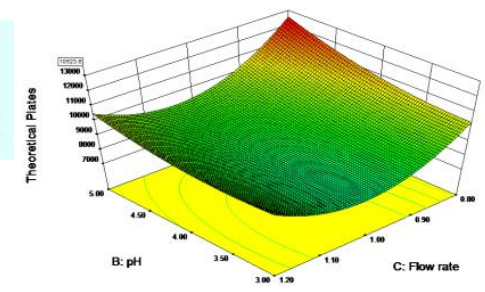

Figure 10(c): Three-dimensional plot for theoretical plates as a function of $\mathrm{pH}$ of buffer and flow rate. Constant factor (amount of buffer)

Fit summary: Quadratic model was suggested by the software

ANOVA: ANOVA of developed CCD model for theoretical plates $\left(\mathrm{Y}_{3}\right)$.

Values of "Prob > F" (p- value) less than 0.0500 indicate model terms are significant. In this case $\mathrm{B}, \mathrm{AC}, \mathrm{B}^{2}$ and $\mathrm{C}^{2}$ are significant model terms. 
Table 22: Significance of $\boldsymbol{p}$ value on model terms of theoretical plates

\begin{tabular}{|l|l|l|l|}
\hline Model terms & $\boldsymbol{p}$ value & Effect of factor & Remarks \\
\hline $\mathrm{A}\left(\mathrm{X}_{1}\right)$ & $\mathbf{0 . 0 0 4 3}$ & -388.5 & Significant \\
\hline $\mathrm{B}\left(\mathrm{X}_{2}\right)$ & 0.4460 & +240.4 & Insignificant \\
\hline $\mathrm{C}\left(\mathrm{X}_{3}\right)$ & 0.3866 & -211 & Insignificant \\
\hline $\mathrm{AB}\left(\mathrm{X}_{1} \mathrm{X}_{2}\right)$ & 0.1936 & -419.2 & Insignificant \\
\hline $\mathrm{AC}\left(\mathrm{X}_{1} \mathrm{X}_{3}\right)$ & 0.3302 & +0.50 & Insignificant \\
\hline $\mathrm{BC}\left(\mathrm{X}_{2} \mathrm{X}_{3}\right)$ & 0.4769 & -78.1 & Insignificant \\
\hline $\mathrm{A}^{2}\left(\mathrm{X}^{2}\right)$ & 0.7040 & -210.2 & Insignificant \\
\hline $\mathrm{B}^{2}\left(\mathrm{X}_{2}\right)$ & 0.4282 & +387.2 & Insignificant \\
\hline $\mathrm{C}^{2}\left(\mathrm{X}_{3}\right)$ & $\mathbf{0 . 0 0 3 0}$ & +73.2 & Significant \\
\hline Overall model & $\mathbf{0 . 0 1 3 4}$ & - & Significant \\
\hline
\end{tabular}

For response $\mathrm{Y}_{3}$, factor $\mathrm{pH}$ of buffer was having synergistic effect with $p$ value 0.4460 . Therefore we can conclude that increment in $\mathrm{pH}$ of buffer was responsible for increment in theoretical plates and thus showed the direct relationship between them.

Combination of amount of ACN and flow rate was responsible for significant increase in theoretical plates with significant $p$ value of 0.0043 . Therefore direct relationship between this combination and response is observed. Exponential terms $\left(\mathrm{X}_{2}^{2}\right)$ and $\left(\mathrm{X}_{3}\right)^{2}$ also showed direct relationship with response having significant $p$ values 0.4282 and 0.0030 respectively. Factor $\mathrm{pH}$ of buffer and its exponential term differs from zero with a great margin therefore they were having more significant effect than other factors. Therefore it is concluded that $\mathrm{pH}$ of buffer and its exponential term was responsible for significant change in response i.e. nos. of theoretical plates. 10,11,12,13,14.

\subsection{Analytical Method Development}

Different mobile phases were investigated to develop the suitable HPLC method for the analysis of Efavirenz in formulations. For the selection of media the criteria employed was sensitivity of the method, ease of sample preparation, miscibility of the drug, cost of solvents and applicability of method to various purposes. Retention time and peak area of EFV in the selected medium at respective wavelengths were determined and compared with the reference standards and formulation also.9,10

\section{Chromatogram of Efavirenz}

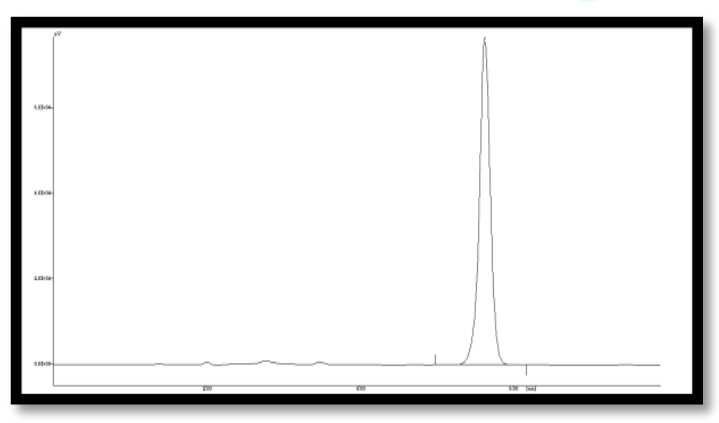

Figure 11: A typical chromatogram of Efavirenz

The applied chromatographic conditions permitted a good separation of Efavirenz in a short retention time of $5.642 \mathrm{~min}$. (Fig.11), no drug decomposition was observed during the analysis. The LC method was validated for the parameters reported below.

\subsection{System suitability study}

According to USP, system suitability tests are integral part of liquid chromatographic methods. Retention time, peak area and number of theoretical plates were calculated for standard solutions. The obtained data was found to be within acceptable limits.

Table 23: System suitability parameters

\begin{tabular}{|l|l|l|}
\hline Sr. No. & Parameters & Observation \\
\hline 1 & Retention time & 5.6 \\
\hline 2 & Peak area & 764985.5 \\
\hline 3 & Theoretical plates & 8182.42 \\
\hline
\end{tabular}

\subsection{Analytical Validation}

\subsubsection{Preparation of Calibration Curve}

Stock solution was prepared using $10 \mathrm{mg}$ of Efavirenz in $10 \mathrm{ml}$ of methanol and further dilutions were done with mobile phase. Solutions were sonicated for $5 \mathrm{~min}$ in Ultrasonic clean bath and manually $20 \mu \mathrm{l}$ was injected through the Rheodyne injector. Five concentrations were taken $5-25 \mu \mathrm{g} / \mathrm{ml}$ for the calibration curve at $292 \mathrm{~nm}$. Linearity graph for Efavirenz is shown in Figure no 12 and Overly spectra of Efavirenz Linearity shown in figure no $13 .^{6}$

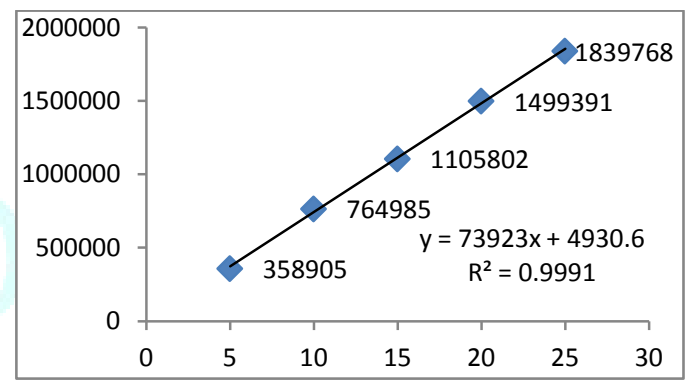

Figure 12: Linearity graph for Efavirenz

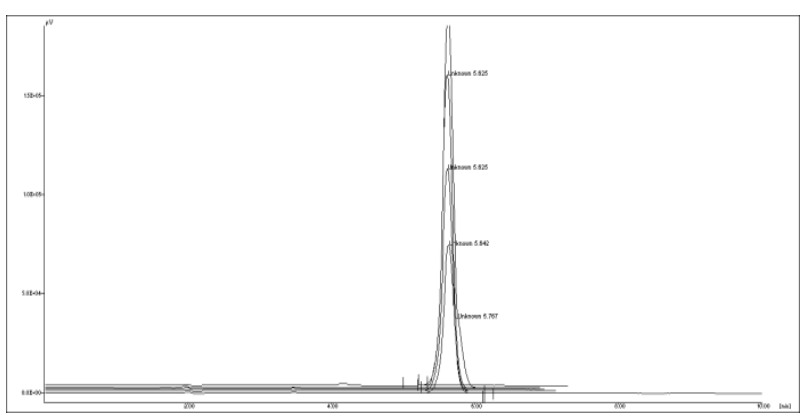

Figure 13: Overly spectra of Efavirenz Linearity

\subsubsection{Selectivity}

10 tab of EFV formulation were triturated in mortar pestle and $35 \mathrm{mg}$ (10 $\mathrm{mg}$ of pure drug) of tab including other excipients was transferred in $10 \mathrm{ml}$ volumetric flask and volume was adjusted with methanol. Peak area and retention time of formulation was compared with the API of the drug. Typical peak of Efavirenz is shown in figure no $14 .^{7}$ 


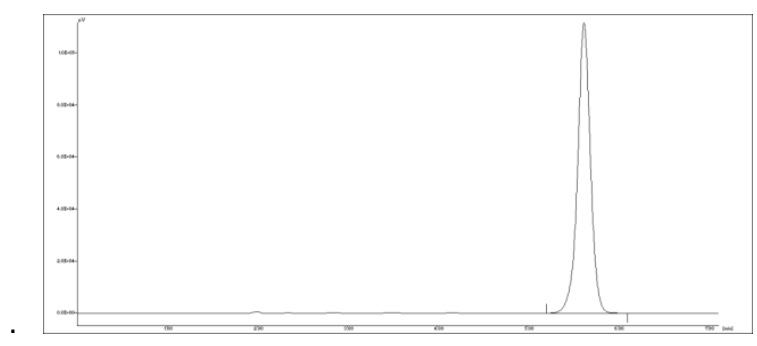

Figure 14: A typical chromatogram of Efavirenz [Concentration 15ug/ml]

\subsubsection{Sensitivity}

The sensitivity of measurement of EFV by use of the proposed method was estimated in terms of the limit of detection (LOD) and the limit of quantification (LOQ). The LOD and LOQ were calculated by the use of signal to noise ratio. In order to estimate the LOD and LOQ values, the blank sample was injected six times and the peak area of this blank was calculated as noise level. The LOD was calculated as three times the noise level, while ten times the noise value gave the LOQ. LOD and LOQ were found to be 0.126 and $0.420 .^{9}$

\subsubsection{Accuracy}

Accuracy of analytical procedure expresses the closeness of agreement between the value which is accepted either as a conventional true value or an accepted reference value and the value found. This is sometimes termed trueness. Accuracy should be established across the specified range of 98-102\%.

For accuracy three concentrations of five replicates were prepared and injected to get the peak area. Results obtained are shown in table 101.11

Table 24: Accuracy results of EFV by RP-HPLC

\begin{tabular}{|l|l|l|l|}
\hline $\begin{array}{l}\text { Concentration } \\
(\mathbf{u g} / \mathbf{m l})\end{array}$ & Peak area & $\begin{array}{l}\text { Concentration Found } \\
(\boldsymbol{\mu g} / \mathbf{m l})\end{array}$ & Accuracy \\
\hline 7 & 276510 & 7.034880 & 100.498296 \\
\hline 17 & 671362.4 & 17.25204161 & 101.4825 \\
\hline 22 & 854793 & 21.99847332 & 99.9930 \\
\hline
\end{tabular}

\subsubsection{Precision}

The precision of an analytical procedure expresses the closeness of agreement (degree of scatter) between the series of measurements obtained from multiple sampling of the same homogeneous sample under the prescribed conditions. Method of precision was accessed on two levels, intra and intermediate precision. Solutions of known concentration were prepared in replicates and were injected to get the peak area. Results obtained are shown in table 99 . 12

Table 25: System Precision results for EFV by RP-HPLC

\begin{tabular}{|l|l|l|l|}
\hline Sr. No. & Concentration $\mathbf{( \mu g} / \mathbf{m l})$ & Intraday precision (Area) & Interday precision (Area) \\
\hline 1 & 20 & 1504212 & 1512836 \\
\hline 2 & 20 & 1504696 & 1516408 \\
\hline 3 & 20 & 1525934 & 1538275 \\
\hline 4 & 20 & 1499974 & 1502956 \\
\hline 5 & 20 & 1506282 & 1519144 \\
\hline 6 & 20 & 1485784 & 1495784 \\
\hline Mean & & 1504480.333 & 1514233.833 \\
\hline Std. Dev. & & 12911.11344 & 14672.11126 \\
\hline \%RSD & & 0.858177615 & 0.968946205 \\
\hline
\end{tabular}

\subsubsection{Specificity}

Chromatogram of EFV showed peak at a retention time of $5.018 \mathrm{~min}$. The mobile phase designed for the method resolved the drug very efficiently; The Retention time of Efavirenz was $5.020 \pm 0.0078 \mathrm{~min}$. The wavelength 292 $\mathrm{nm}$ was selected for detection because; it resulted in better detection sensitivity for the drug. The peak for Efavirenz from the tablet formulations was identified by comparing its retention time and Area of peak with those of standard Efavirenz. Recovery was found to be $99.28 \pm 0.004$. The result was shown in table 107.13

Table 26: Recovery results of EFV by RP-HPLC

\begin{tabular}{|l|l|l|l|l|}
\hline Sample & Label Claim mg & Amount found* $(\mathbf{m g})$ & Recovery \pm SD* $(\%)$ & Retention time \\
\hline Tab & 600 & 599.96 & $99.28 \pm 0.004$ & $5.6 \pm 0.0078 \mathrm{~min}$ \\
\hline
\end{tabular}

\subsubsection{Repeatability}

Demonstration of precision was done under two categories. The injection repeatability (System Precision) was assessed by using six injections of the standard solution of EFV and the \% RSD of the replicate injections was calculated. Results obtained are shown in table 100.14 
Table 27: Repeatability results for EFV by RP-HPLC

\begin{tabular}{|l|l|l|}
\hline Sr. no & $\begin{array}{l}\text { Concentration } \\
\boldsymbol{\mu g} / \mathbf{m l}\end{array}$ & Peak Area \\
\hline 1 & 20 & 1490913 \\
\hline 2 & 20 & 1497975 \\
\hline 3 & 20 & 1499391 \\
\hline 4 & 20 & 1516768 \\
\hline 5 & 20 & 1499974 \\
\hline 6 & 20 & 1516768 \\
\hline Mean & & 1503631.5 \\
\hline Std. Dev & & 10679.4255 \\
\hline \% RSD & & 0.710242204 \\
\hline
\end{tabular}

\subsubsection{Robustness}

Robustness is a measure of capacity of a method to remain unaffected by small, but deliberate variations in the method conditions, and is indications of the reliability of the method. A method is robust, if it is unaffected by small changes in operating conditions. To determine the robustness of this method, the experimental conditions were deliberately altered at three different levels and retention time and chromatographic response were evaluated. One factor at a time was changed to study the effect. Variation of mobile phase composition (72:28 $\mathrm{v} / \mathrm{v})$, flow rate by $1 \mathrm{ml} / \mathrm{min}(0.9$ and $1.1 \mathrm{ml} / \mathrm{min})$, Variation of wavelength by $247 \mathrm{~nm}(245 \mathrm{~nm}$ and $247 \mathrm{~nm})$, Variations in pH by 5 (4.8 and 5.2) had no significant effect on the retention time and chromatographic response of 10 $\mu \mathrm{g} / \mathrm{ml}$ solution, indicating that the method was robust, The result was shown in table 102, 103,104 and 105.6,7.

\section{Mobile Phase}

Table 28: Results of Robustness (Mobile Phase) for EFV by RP-HPLC

\begin{tabular}{|l|l|l|}
\hline Buffer & Acetonitrile & Retention time \\
\hline 30 & 70 & 5.9 \\
\hline 28 & 72 & 5.6 \\
\hline 26 & 74 & 5.3 \\
\hline & \%RSD & 1.8 \\
\hline
\end{tabular}

\section{Wavelength}

Table 29: Results of Robustness (Wavelength) for EFV by RP-HPLC

\begin{tabular}{|l|l|}
\hline Wavelength & Peak area \\
\hline 245 & 742144.500 \\
\hline 247 & 735436.709 \\
\hline 249 & 724684.750 \\
\hline \%RSD & 1.2 \\
\hline
\end{tabular}

\section{Flow rate}

Table 30: Results of Robustness (Flow rate) for EFV by RP-HPLC

\begin{tabular}{|l|l|}
\hline Flow rate & Retention time \\
\hline $0.9 \mathrm{ml} / \mathrm{min}$ & 6.1 \\
\hline $1 \mathrm{ml} / \mathrm{min}$ & 5.6 \\
\hline $1.1 \mathrm{ml} / \mathrm{min}$ & 5.1 \\
\hline$\% \mathrm{RSD}$ & 1.7 \\
\hline
\end{tabular}

\section{pH of mobile phase}

Table 31: Results of Robustness (pH) for EFV by RP-HPLC

\begin{tabular}{|l|l|l|}
\hline Sr. No & pH & Peak area \\
\hline 1 & 4.8 & 742144.500 \\
\hline 2 & 5 & 765436.709 \\
\hline 3 & 5.2 & 784684.750 \\
\hline & \%RSD & 1.2 \\
\hline
\end{tabular}

\subsubsection{Recovery}

The recovery of the method was determined by use of standard additions at three different levels, i.e. multiplelevel recovery studies. Preanalysed samples of EFV were spiked with extra 80, 100 and $120 \%$ of the standard EFV and the mixtures were reanalysed by the proposed method and the \% recovery was determined. Values were found to be within the limits and are presented in table 106 . 2,3 .

Table 32: Recovery results of EFV by RP-HPLC

\begin{tabular}{|l|l|l|l|l|}
\hline Sr. No & $\begin{array}{l}\text { Amount of } \\
\text { sample } \\
(\boldsymbol{\mu g} / \mathbf{m l})\end{array}$ & $\begin{array}{l}\text { Amount of drug } \\
\text { added }(\boldsymbol{\mu g} / \mathbf{m l})\end{array}$ & $\begin{array}{l}\text { Amount } \\
\text { Recovered* } \\
(\mu \mathrm{g} / \mathbf{m l})\end{array}$ & $\begin{array}{l}\% \text { Recovery } \pm \\
\text { SD }^{*}\end{array}$ \\
\hline 1 & 10 & 8 & 7.96 & $98.88 \pm 0.31$ \\
\hline 2 & 10 & 10 & 9.88 & $99.01 \pm 0.58$ \\
\hline 3 & 10 & 12 & 11.72 & $98.46 \pm 0.41$ \\
\hline
\end{tabular}

\section{SUMMARY AND CONCLUSION}

\subsection{Determination of Efavirenz by HPLC.}

In this project, as per our objective RP-HPLC method was developed by implementing QbD methodology (RSM and 3 level factorial design) on analytical column- reversed phase Waters XBridge C18 $(250 \mathrm{~mm} \times 4.6 \mathrm{~mm} \times 5 \mu \mathrm{m})$ with mobile phase Amonium format: Acetonitrile (28:72 v/v). The flow rate used was $1 \mathrm{~mL} / \mathrm{min}$ and UV detection was carried out at $247 \mathrm{~nm}$. The retention time for Efavirenz was found to be 5.6 min. Before method optimization, screening studies were carried out on different mobile phases, varying composition and $\mathrm{pH}$. Based on the results obtained from these studies, suitable mobile phase with appropriate composition and $\mathrm{pH}$

ISSN: 2250-1177 was selected and utilized for method development using QbD methodology.

The RP-HPLC method developed for estimation of Efavirenz was validated as per ICH guidelines using various parameters.

Linearity for the drug by the proposed method was determined to study its ability to elicit test results which are directly proportional to the concentration of analyte in the sample response and was found to be in concentration range 5 to $25 \mu \mathrm{g} / \mathrm{mL}(\mathrm{R} 2=0.998)$.

The limit of detection (LOD) and limit of quantitation (LOQ) was established at a signal-to-noise ratio. LOD and LOQ were calculated as $3.3 \times \delta / S$ and $10 \times \delta / S$ respectively as per ICH CODEN (USA): JDDTAO 
guidelines, where $\delta$ is the standard deviation of the response ( $y$-intercept) and $S$ is the slope of the calibration plot. LOD was found to be $0.126 \mu \mathrm{g} / \mathrm{mL}$ and LOQ was found to 0.420 $\mu \mathrm{g} / \mathrm{mL}$

System suitability test ensures that the analytical system is working properly and can give accurate and precise results. System suitability tests includes resolution, tailing factor, number of theoretical plates, capacity factor etc. The results of all system suitability parameters were acceptable in their limits defined by official guidelines.

The proposed high-performance liquid chromatographic method has also been evaluated over the accuracy, precision and robustness and proved to be convenient and effective for the quality control of Efavirenz. Developed method was found simple and cost effective for the quality control of Efavirenz.

Moreover, the lower solvent consumption along with the short analytical run time of 10.0 min leads to a cost effective and environmentally friendly chromatographic procedure. Thus, the proposed methodology is rapid, selective, requires a simple sample preparation procedure, and represents a good procedure for Efavirenz.

Table 33: Results of the quantitative determination of Efavirenz.

\begin{tabular}{|l|l|l|}
\hline $\begin{array}{l}\text { Sr. } \\
\text { no }\end{array}$ & Parameters & HPLC observations \\
\hline 1 & Concentration range $(\mu \mathrm{g} \mathrm{mL}-1)$ & $5-25$ \\
\hline 2 & Regression equation & $\mathrm{y}=73923 \mathrm{x}+4930.6$ \\
\hline 3 & Correlation coefficient & 0.998 \\
\hline 4 & LOD $(\mu \mathrm{g} \mathrm{mL}-1)$ & 0.126 \\
\hline 5 & LOQ $(\mu \mathrm{g} \mathrm{mL}-1)$ & 0.420 \\
\hline
\end{tabular}

\section{FUTURE SCOPE}

QbD methodology can be applied for Bioanalytical method development and its validation for estimation of EFV.

QbD methodology can be applied for stability indicating studies of EFV.

\section{REFERENCES}

1. Udaykumar RB, Stability- indicating HPLC method for the determination of efavirenz in bulk drug and in pharmaceutical dosage form, African Journal of Pharmacy and Pharmacology, 2009; 3(12):643-650.

2. Savie IM, Experimental design to quality by design in pharmaceutical legislation, Accreditation and Quality Assurance, 2012; 17:627-633.

3. Rajkotwala AS, Shaikh SS, QbD Approach to Analytical Method Development and Validation of Piracetam By HPLC, World Journal of Pharmacy and Pharmaceutical Sciences, 2016; 5(5):1771-1784

4. Venkata RY, Pal KA, Validated stability indicating analytical method and invitro dissolution studies of efavirenz formulation by RP-HPLC, International Journal of Pharmacy and Pharmaceutical Sciences, 2012; 4(15):572-576.

5. Appala R, Simultaneous estimation of tenofovir disoproxil, emtricitabine and efavirenz in tablet dosage form by RP-HPLC, Oriental Journal of Chemistry, 2008; 24(2):645-650.

6. Brajendra SR, Method Development and Validation Of RP-HPLC Method In The Detection Of Efavirenz In Bulk Drug And Tablet Formulation, International Research Journal of Pharmacy, 2012; 3(7):297-300.

7. Behnken DW, Some new three level designs for the study of quantitative variables, Technometrics, 1960; 2:455-475.

8. Bruns RE, Scarminio IS, Neto BB, Statistical designChemometrics, Amsterdam, 2006:338-350.

9. Bhavsar SA, RP-HPLC method for simultaneous estimation of tenofovir disoproxil fumarate, lamivudine, and efavirenz in combined tablet dosage form, Pharmaceutical Methods, 2012; 3(2):73-78.

10. Campana AM, Rodriguez, LC, Gonzalez, AL. Sequential response surface methodology for multioptimization in analytical with three-variable Doehlert designs, Analytical Chemica Acta, 1997; 348:237-246.

11. Doehlert, DH, Uniform shell designs, Journal of the Royal Statistical Society, 1970; 19:231-239.

12. Ermer J, Hans-JP, Validation in pharmaceutical analysis Part II: central importance of precision to establish acceptance criteria and for verifying and improving the quality of analytical data, Journal of Pharmaceutical and Biomedical Analysis, 2005; 37:859-870.

13. Fabre H, Robustness testing in liquid chromatography and capillary electrophoresis, Journal of Pharmaceutical and Biomedical Analysis, 1996; (14):1125-1132.

14. Gilmour SG, Response surface designs for experiments in bioprocessing' Biometrics, 2006; (62):323-331. 